\title{
Philonsorbonne
}

1 | 2007

Année 2006-2007

\section{Le moi peut-il être sauvé ? La subjectivité, de Mach au premier Wittgenstein}

Sabine PLAUD

\section{OpenEdition}

1 Journals

Édition électronique

URL : https://journals.openedition.org/philonsorbonne/123

DOI : $10.4000 /$ philonsorbonne.123

ISSN : $2270-7336$

Éditeur

Publications de la Sorbonne

\section{Édition imprimée}

Date de publication : 15 novembre 2007

Pagination : 85-102

ISBN : 978-2-85944-594-2

ISSN : 1255-183X

\section{Référence électronique}

Sabine PLAUD, «Le moi peut-il être sauvé ? La subjectivité, de Mach au premier Wittgenstein », Philonsorbonne [En ligne], 1 | 2007, mis en ligne le 27 janvier 2013, consulté le 08 juin 2021. URL http://journals.openedition.org/philonsorbonne/123; DOI : https://doi.org/10.4000/philonsorbonne. 123

(c) Tous droits réservés 


\title{
Le moi peut-il être sauvé ? \\ La subjectivité, de Mach au premier Wittgenstein
}

\author{
Sabine Plaud
}

Bien que l'histoire de la philosophie soit jalonnée de questions portant sur la nature de la subjectivité, cette interrogation prendra une importance toute particulière au tout début $\mathrm{du} X \mathrm{X}^{\mathrm{e}}$ siècle au sein de la Modernité viennoise $^{1}$. Nous étudierons ici notamment la façon dont cette question a été abordée par Ernst Mach et Ludwig Wittgenstein, deux Viennois qui ont en commun une même attitude paradoxale à l'égard de la subjectivité. D'une part, tous deux semblent en effet placer le sujet au cœur de leurs philosophies respectives, en allant jusqu'à développer des thèses en apparence idéalistes ou solipsistes ; mais d'autre part, tous deux semblent également soutenir des thèses qui vont au contraire jusqu'à éliminer un sujet dont on affirmera qu'il «ne peut être sauvé ».

C'est cette contradiction apparente que nous nous proposons d'examiner ici. Afin de la résoudre, nous nous pencherons sur la distinction qu'établit le premier Wittgenstein entre deux types de sujet : un sujet «psychologique » et un sujet «métaphysique ». Nous verrons que cette dualité est la clé qui nous permet de comprendre l'attitude apparemment ambiguë de Wittgenstein à l'égard de la subjectivité, et ce parce qu'elle permet d'affirmer sans contradiction tout à la fois la primauté et la disparition du sujet. En admettant que le sujet psychologique ne puisse être sauvé, il n'en sera pas moins, chez Wittgenstein, possible de sauver le sujet philosophique. En ce sens, nous défendrons l'idée que la philosophie du premier Wittgenstein parvient à échapper à cette crise interprétative qui menaçait la pensée de son prédécesseur viennois.

1. Cet article, qui reprend les thèmes abordés dans notre travail de DEA, est la traduction d'une intervention prononcée en avril 2006 à la New School for Social Research de New York sous le titre «Must the Ego Really be Given up ? Subjectivity from Mach to the Early Wittgenstein ». 
Pourtant, à quoi Wittgenstein se réfère-t-il exactement quand il évoque ce sujet philosophique ? S'agit-il d'un sujet transcendantal de type kantien, d'un sujet qui serait une condition de possibilité tant du monde que de l'unité de nos représentations? Nous montrerons que si une telle interprétation est séduisante, elle est cependant difficile à tenir jusqu' au bout. Nous verrons au contraire que si le sujet philosophique wittgensteinien peut être qualifié de «transcendantal », il ne le sera cependant pas en un sens kantien : que ce sujet soit transcendantal, ici, signifie bien plutôt qu'il est le sujet d'une volonté éthique.

Nous commencerons donc par présenter l'attitude paradoxale de Mach à l'égard de la subjectivité, avant de comparer celle-ci avec la façon ambiguë dont le premier Wittgenstein aborde cette même question. Enfin, nous essaierons de résoudre cette ambiguité en examinant la distinction wittgensteinienne entre sujet psychologique et sujet philosophique.

\section{Le paradoxe de la subjectivité chez Ernst Mach : de l'idéalisme à l'élimination du sujet}

\section{Idéalisme et phénoménisme : la primauté du Moi}

Ernst Mach (1838-1916) n'était pas philosophe mais physicien. Bien plus, il rejetait même ouvertement le titre de philosophe en affirmant qu' «il n'y a pas de philosophie machienne $»^{2}$. Pourtant, il y a sans aucun doute une contribution de Mach à la question philosophique de la subjectivité : cette contribution est une conséquence de la conception générale de la réalité qu'il présente dans les «Remarques préliminaires anti-métaphysiques » de son ouvrage de 1886 intitulé L'Analyse des sensations. Mach y développe en effet une ontologie selon laquelle la réalité consiste en «éléments » ou en «sensations ». Ces dernières sont à leur tour rassemblées en complexes, et ce sont ces complexes que nous avons coutume de considérer comme des « choses » ou des « objets ». Ces « choses » ou « objets » n'ont pourtant pas d'unité propre : leur unité apparente ne leur est conférée que par un acte de synthèse accompli par un sujet de la perception qui découpe le réel en fonction de ses besoins. En d'autres termes :

La chose, le corps, la matière, ne sont [...] rien en dehors de la connexion des éléments, des couleurs, des sons $[\ldots]^{3}$

Cette ontologie machienne ne se présente pas nécessairement d'emblée comme une forme d'idéalisme. En effet, ces éléments machiens sont en

2. Mach, Erkenntnis und Irrtum, Skizzen zu eine Psychologie der Forschung, Leipzig, 1905. Tr. M. Dufour, La Connaissance et l'erreur, Paris, préface à la $1^{\text {ère } e ́ d i t i o n, ~ n o t e ~} 1, \mathrm{p}$. XXIII.

3. Mach, Die Analyse der Empfindungen, Iena, G. Fischer, 1886, tr. F. Eggens et J.-M. Monnoyer : L'Analyse des sensations (AS), Nîmes, J. Chambon, 1996, p. 11. 
apparence de trois types dont un seulement se rapporte à la conscience. On distingue ainsi entre les $\mathrm{A}, \mathrm{B}, \mathrm{C}$, qui concernent la réalité physique en général (c'est-à-dire "les complexes de couleurs, de sons, etc. que nous appelons habituellement des corps $»^{4}$ ) ; les K, L, M, qui se rapportent au « complexe qui se nomme notre corps » et «que certaines particularités ont rendu remarquable $»^{5}$. Enfin, on trouve les $\alpha, \beta, \gamma$, qui constituent la conscience proprement dite. Pourtant, l'aspect idéaliste de la pensée de Mach apparaît à ceci que, dans chacun de ces trois cas, les éléments sont en même temps des sensations. C'est pourquoi les constituants ultimes de la réalité ne sont rien d'autre que les données premières de la perception, de sorte que l'ontologie de Mach peut être considérée comme une forme de phénoménisme selon lequel «le monde n'est constitué que de nos sensations $»$.

Un tel phénoménisme semble bien appuyer une lecture subjectiviste ou solipsiste de la pensée machienne. $\mathrm{Si}$, en effet, la réalité n'est rien de plus qu'un phénomène, alors il n'y a rien de tel qu'un monde objectif ou indépendant du sujet. On peut rappeler, par exemple, cette célèbre critique adressée par Lénine à Mach dans son ouvrage intitulé Matérialisme et empiriocriticisme, critique par laquelle il reproche à Mach d'avoir dissimulé une régression vers une métaphysique idéaliste sous le masque d'un programme positiviste et matérialiste :

Aucun subterfuge, aucun sophisme [...] ne voileront ce fait indiscutable et bien clair que la doctrine d'Ernst Mach, suivant laquelle les choses sont des complexes de sensations, n'est qu'idéalisme subjectif, que rabâchage de la théorie de Berkeley. Si, d'après Mach, les corps sont des «complexes de sensations » ou, comme disait Berkeley, des «combinaisons de sensations », il s'ensuit nécessairement que le monde entier n'est que représentation? ${ }^{7}$.

Bien entendu, la justesse d'un tel reproche peut être contestée puisque Mach refusait d'être considéré comme un idéaliste au sens berkeleyen, de même qu'il rejetait vigoureusement un solipsisme qu'il allait jusqu'à qualifier de «monstruosité ${ }^{8}$. Cependant, ces lectures solipsistes de la pensée machienne ont le mérite de mettre en lumière la façon dont Mach assigne effectivement au sujet une incontestable primauté. Nous allons à présent examiner la façon dont cette approche subjectiviste se heurte à un autre aspect de la pensée de Mach qui nous met quant à lui face à une élimination radicale de la subjectivité.

4. AS, p. 13.

5. Ibid.

6. Ibid., p. 16.

7. Lénine, Matérialisme et empiriocriticisme, Pékin, Éditions en langues étrangères, 1974, p. 37.

8. Ibid., p. 28. 


\section{L'élimination de la subjectivité}

De même que l'ontologie machienne implique une déconstruction des « objets », de même elle implique une déconstruction des «sujets ». Il y a en effet trois raisons principales qui conduisent l'ontologie de Mach à une critique sceptique de la notion de sujet. La première de ces raisons est que si la réalité consiste essentiellement en éléments, alors tous ces éléments partagent une seule et même nature : en conséquence, il n'y a pas de différence essentielle entre le Moi et le reste du monde. Certes, il y a bien une différence relative entre les trois catégories de sensations, mais cette différence est au mieux une différence fonctionnelle et nullement une différence essentielle. En d'autres termes, un seul et même complexe d'éléments peut être alternativement considéré comme physique ou psychique. C'est pourquoi, selon Mach :

Sur cette voie, nous ne rencontrons pas de fossé entre corps et sensations, entre intérieur et extérieur, entre monde matériel et monde physique (...). Tous les éléments, ABC, KLM, s'agrègent en une seule masse cohérente?.

Une telle conception est ce qui est communément désigné par l'expression de «monisme neutre». Selon le monisme neutre, la réalité n'est, en elle-même, ni physique ni psychique mais peut se voir attribuer successivement ces deux déterminations. Le Moi lui-même n'est donc pas séparé du reste de la réalité physique, mais y est au contraire intégré. On peut mentionner, par exemple, cette soudaine prise de conscience par Mach de l'unité de son Moi avec le reste de la nature :

Par un beau jour d'été en plein air, le monde m'est soudain apparu comme formant, avec mon propre Moi, une seule masse complexe de sensations, à la seule différence que cette complexité était plus grande dans le $\mathrm{Moi}^{10}$.

Et puisque le Moi n'est qu'une partie de la nature, alors la psychologie, en tant qu'étude du Moi, devient à son tour une simple science empirique sur le même plan que la physique ou que la biologie.

Mais il y a une deuxième raison pour laquelle l'ontologie machienne est vouée à ruiner le concept de sujet. Dans la mesure où il n'y a, en général, pas de « choses », alors il n'y a, en particulier, pas de «choses pensantes ». Les conclusions que Mach tirait à propos des objets se voient ainsi appliquées aux sujets, et l'identité du moi s'avère être à son tour une identité purement fictive et fonctionnelle : «un moi isolé n'existe pas plus qu'une chose isolée. Tous deux sont des fictions provisoires du même type $»^{11}$.

9. $A S$, p. 20.

10. Ibid., p. 17.

11. La connaissance et l'erreur, p. 15. 
C'est là précisément ce qui nous conduit à la troisième raison de cette élimination machienne de la subjectivité. Puisque le Moi n'a pas d'unité déterminée, alors il devient impossible de tracer une frontière nette entre le sujet et ce qui n'est pas lui. Si le sujet n'est rien d'autre qu'un ensemble de représentations, et si le monde lui-même consiste en représentations, alors le sujet se confond avec le monde en général et ne possède pas d'identité propre : «le Moi peut être élargi au point d'inclure le monde entier ${ }^{12}$.

Bien entendu, cette réévaluation par Mach de l'unité du sujet implique une réfutation de la notion d'identité personnelle. La conscience fait ainsi l'objet de constantes interruptions (sommeil, amnésie...), de divisions (rêves, dédoublements de personnalité...), etc. Le prétendu «Moi » est donc soumis à un perpétuel changement sans qu'il y ait un quelconque substrat qui compense ce changement par sa stabilité : le Moi n'est jamais identique à lui-même. C'est pourquoi Mach fait sien ce célèbre aphorisme de Lichtenberg dirigé contre le cogito cartésien : «on devrait dire : ça pense », comme on dit, à propos de l'éclair : ça luit (es blitzet) $»^{13}$. Cette idée sera transposée par le physicien dans une formulation qui deviendra presque un slogan, une devise emblématique de la Modernité viennoise : «le Moi ne peut être sauvé », «das Ich ist unrettbar $»^{14}$.

Une telle approche de la subjectivité a bien entendu des conséquences morales immédiates, puisque le sujet ainsi éliminé est également le sujet de la volonté, de la responsabilité, de l'éthique. Certes, l'intention de Mach n'est pas de produire un effondrement des fondements de la morale : il n'en reste pas moins qu'à défaut d'être tirées par Mach lui-même, de telles conséquences sceptiques seront tirées quelques années plus tard par d'autres auteurs viennois, notamment par Robert Musil dont l'idée d'un «homme sans qualités », dans laquelle on peut lire l'un des aspects du malaise moderne, porte certainement des traces de cette dissolution machienne du sujet.

Mais c'est là précisément ce qui nous conduit à notre problème principal. D'une part, Mach soutient que le monde n'a pas de réalité à moins d'être perçu par un sujet. D'autre part, il affirme que le Moi doit être éliminé. Mais comment cette élimination machienne du Moi peut-elle s'accorder avec les considérations solipsistes de ce même auteur ? Comment peut-on tout à la fois défendre une position idéaliste et affirmer qu'il n'y a rien de tel qu'un sujet de la conscience ${ }^{15}$ ? Nous allons à présent essayer de

12. AS, p. 17.

13. AS, p. 30 .

14. AS, p. 27.

15. Cette difficulté était déjà soulignée par Philipp Frank qui écrivait, dans un article consacré à la pensée de Mach, que «d'une part, Mach est présenté comme l'adversaire le plus radical de toute tentative d'introduire dans la science des facteurs ayant la moindre nuance de spiritualisme. [...] D'autre part, on nous dit que, selon Mach, notre monde consiste entièrement en perceptions ou en complexes de perceptions ; [...] pour cette raison, Mach s'est vu proclamer le champion de la philosophie idéaliste au sein de la science moderne » («Ernst Mach and the Unity of Science », in Erkenntnis, 7, 1938, p. 235-236). 
trouver une solution à ce problème en examinant les échos de cette pensée machienne dans l'œuvre du premier Wittgenstein.

\section{Le paradoxe de la subjectivité chez le premier Wittgenstein : du solipsisme au réalisme pur}

En dépit des nombreuses critiques que Wittgenstein devait adresser à Mach, on peut trouver un grand nombre de points communs à ces deux penseurs : conception des propositions comme modèles des faits, rejet de la métaphysique et de ses faux problèmes... Nous allons nous pencher en particulier sur le rapport qui existe entre l'ambiguïté machienne à l'égard de la subjectivité et le traitement de cette même question dans la section $5.6 \mathrm{du}$ Tractatus logico-philosophicus (TLP). Cette section commence en effet par suggérer une conception idéaliste ou solipsiste du sujet, avant de s'achever sur une élimination du Moi qui n'est pas sans évoquer sa dissolution machienne. En ce sens, cette section semble bien contenir la même ambivalence que celle qui était contenue dans l'Analyse des sensations de Mach. Nous allons donc examiner successivement ces deux étapes.

\section{«Ce que le solipsisme veut dire est tout à fait correct »}

La proposition inaugurale de la section 5.6 est celle qui affirme que « les limites (Grenzen) de mon langage sont les limites de mon monde ». Cette assertion est caractéristique de ce que l'on désigne en général comme le solipsisme du premier Wittgenstein. Dans la mesure où cette proposition n'est pas immédiatement claire, on peut essayer de clarifier sa signification de la façon suivante. Selon Wittgenstein, la totalité des propositions (le langage) correspond à la totalité des états de choses (au monde). C'est pourquoi «la logique remplit le monde ${ }^{16}$, de sorte que les limites du langage s'avèrent être également celles du monde. Pourtant, Wittgenstein ne se contente pas d'affirmer l'existence d'une coïncidence entre langage et monde en général, mais il affirme celle d'une coïncidence entre mon langage et mon monde. En cela, il défend une approche apparemment solipsiste, approche qu'il explicitera à la proposition 5.621 en écrivant que «le monde et la vie ne font qu'un », ainsi qu'à la proposition 5.63 où l'on pourra lire que « [j]e suis mon monde. (le microcosme)». Ce microcosme n'est autre qu'un monde en miniature, que le petit monde du sujet qui s'avère être identique au macrocosme, au monde en général. Cet aspect du Tractatus peut donc être considéré comme une version de la thèse machienne selon laquelle «le monde n'est constitué que de nos sensations ».

Bien plus, Wittgenstein fait lui-même explicitement allusion à ses propres présuppositions solipsistes en écrivant à la proposition 5.62 que cette

16. L. Wittgenstein, Tractatus logico-philosophicus (TLP), 5.61, tr. G.-G. Granger, Paris, Gallimard, 1993. 
coïncidence entre monde et langage «fournit la clé » pour décider «dans quelle mesure le solipsisme est une vérité ». Sa conclusion est que «ce que le solipsisme veut dire est tout à fait correct ». Il semble donc véritablement y avoir un solipsisme du premier Wittgenstein, lequel peut être caractérisé comme un «solipsisme linguistique».

\section{Aux origines du solipsisme : les Carnets 1914-1916}

Le solipsisme dont il est ici question est en réalité un thème précoce de la philosophie de Wittgenstein, thème dont on peut rechercher les origines dans les Carnets 1914-1916. Dans ces écrits, Wittgenstein ne se contentait pas de défendre la thèse solipsiste classique selon laquelle l'existence du monde dépend d'un sujet, il allait jusqu'à affirmer que ce sujet est le seul sujet, et que son âme se confond avec l'âme du monde :

Il n'y a qu'une seule âme du monde, que je nomme, par préférence, mon âme et conformément à laquelle seule je conçois ce que je nomme l'âme des autres $^{17}$.

Ce Moi est ainsi, dans les Carnets, explicitement désigné comme centre du monde des perceptions, de la conscience, de la volonté. C'est pourquoi Wittgenstein évoque «ce centre du monde, que je nomme le Je et qui est le support de l'éthique ${ }^{18}$. En tant que centre de son monde, le Moi est par conséquent la mesure de toutes choses, l'instance qui fixe toutes les valeurs : « $J$ 'ai à juger le monde, à mesurer les choses ${ }^{19}$, «le bien et le mal n'apparaissent que par le sujet ${ }^{20}$. Pourtant, ce Moi ne s'en tient pas à cette position centrale : il se tient également aux limites du monde. C'est là du moins ce qui émerge de certains passages dans lesquels on peut lire, par exemple, que «le sujet n'appartient pas au monde mais est une limite du monde ${ }^{21}$. C'est pourquoi les limites temporelles du monde ne sont rien d'autre que celles qui lui sont imposées par la vie du sujet : «Dans la mort, le monde ne s'altère pas, il cesse d'exister ${ }^{22}$.

Il semble donc que les Carnets nous rapportent un moment de la pensée de Wittgenstein où celui-ci adhérait résolument à une approche solipsiste de la subjectivité. C'est cette approche dont on trouve les échos au début de la section 5.6 du $T L P$, bien qu'elle soit présentée sous une forme plus sobre qu'elle ne l'était pendant la Première Guerre Mondiale. Mais nous allons voir à présent que cette même section 5.6 nous présente, immédiatement

17. L. Wittgenstein, Carnets 1914-1916, tr. G.-G. Granger, Paris, Gallimard, 1971, 23-5-15.

18. Carnets, 5-8-16.

19. Ibid., 2-9-16.

20. Ibid., 2-8-16.

21. Ibid., 2-8-16.

22. Ibid., 5-7-16. 
après ces réflexions solipsistes, des affirmations qui témoignent au contraire d'un scepticisme radical à l'égard de la notion de sujet.

\section{«Il n'y a pas de sujet de la pensée, de la représentation »}

Le Tractatus renferme un paradoxe qui évoque en tout point le paradoxe machien. On peut en effet récapituler la gradation à laquelle procède le Tractatus à la section 5.6 : on commence par affirmer que les limites de mon langage sont les limites de mon monde, puis on en conclut que le monde et la vie sont un et que je suis mon monde, conclusion qui semble être l'expression d'un solipsisme radical. Pourtant, comme c'était déjà le cas chez Mach, ce solipsisme présente un singulier contraste avec la disparition du Moi qui se produit dans les propositions suivantes. Ainsi, immédiatement après avoir écrit que «[j]e suis mon monde», Wittgenstein affirme abruptement qu' «il n'y a pas de sujet de la pensée, de la représentation $»^{23}$. Ce rejet absolu du sujet cartésien est alors justifié par l'expérience de pensée suivante :

Si j'écrivais un livre intitulé Le monde tel que je l'ai trouvé, je devrais y faire aussi un rapport sur mon corps, et dire quels sont les membres qui sont soumis à ma volonté, lesquels n'y sont pas soumis etc. Ce qui est en réalité une méthode pour isoler le sujet, ou plutôt pour montrer que, en un sens important, il n'y a pas de sujet : car c'est de lui seulement qu'il ne pourrait être question dans ce livre.

L'ouvrage en question contiendrait donc la totalité de ce qui peut être dit : mais il ne saurait contenir une description de son propre auteur, puisque celui-ci «n'appartient pas au monde, mais est une limite du monde ${ }^{24}$. D'une manière analogue, le sujet qui voit et décrit le monde n'a aucune réalité dans le monde et peut par conséquent être éliminé.

Cette idée est encore illustrée à la proposition 5.633 au moyen d'une analogie selon laquelle le sujet est à son monde ce que l'œil est au champ visuel :

Tu dirais qu' il en est ici tout à fait comme de l'œil et du champ visuel. Mais l'œil, en réalité, tu ne le vois pas .

On peut considérer cette image du Tractatus comme un écho à ce passage de l'Analyse des sensations dans lequel Mach s'interrogeait sur la forme que prend la perception du champ visuel. Mach agrémentait en effet son analyse d'un dessin resté célèbre censé représenter « le champ visuel tel que je l'ai trouvé », c'est-à-dire le champ visuel en tant qu'il est dessiné par un sujet qui ferme l'œil droit ${ }^{25}$. Dans ce dessin, on voit le reste de la pièce en 
perspective, on voit le corps propre du dessinateur étendu sur un sofa, et on voit même une partie de son nez, un bout de sa moustache; mais la seule chose que l'on ne saurait dessiner, c'est bien l'œeil du dessinateur :

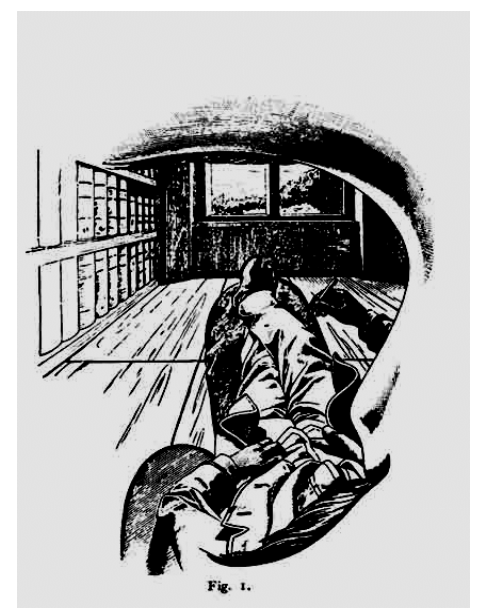

La particularité du champ visuel est donc le fait qu'il soit vu par un œil qui ne se voit pas lui-même. Chez Mach, comme plus tard chez Wittgenstein, au lieu de dire «je vois», on devrait dire «ça voit».

Or la conclusion de Mach dans ce passage était une conclusion antisolipsiste : le Moi est selon lui intégré au reste du monde et ne saurait prétendre occuper une position privilégiée dans celui-ci. Wittgenstein, quant à lui, radicalise ces conclusions anti-solipsistes lorsqu'il applique au rapport sujet-réalité ces considérations sur le champ visuel ${ }^{26}$. Ce renoncement au solipsisme est exprimé très clairement à la proposition 5.64 où il écrit que « le solipsisme, s’il est rigoureusement développé, coïncide avec le réalisme pur»: ce «réalisme pur» n'est en effet rien d'autre que la thèse diamétralement opposée au solipsisme, celle qui veut le monde existe indépendamment de tout sujet. On ne s'étonnera donc pas de trouver, à la fin de la section 5.6, un équivalent wittgensteinien à la thèse machienne selon laquelle «le Moi ne peut être sauvé »:

Le Je du solipsisme se réduit à être un point sans étendue, et il reste la réalité qui lui est coordonnée ${ }^{27}$.

26. Quelques années plus tard, au $§ 213$ des Remarques Philosophiques, Wittgenstein s'opposera même explicitement à ce schéma machien auquel il reprochera de nous reconduire, sous couvert d'une critique du solipsisme, à une conception du champ visuel comme quelque chose de centré et de limité, ce qui est en réalité typiquement une approche solipsiste de la perception. Sur ce désaveu du schéma machien par Wittgenstein on consultera l'article de Jérôme Sackur intitulé «Théorie de la connaissance et solipsisme» (in Wittgenstein: Les mots de l'esprit, dir. Ch. Chauviré, S. Laugier et J.-J. Rosat, Paris, Vrin, 2002).

27. TLP 5.634 . 
Ainsi, on se souvient de ce que, chez Mach, « le Moi peut être élargi au point d'inclure le monde entier »; chez Wittgenstein, au contraire, le Moi rétrécit au point de disparaître entièrement : mais dans les deux cas, on est face à une disparition du sujet qui nous reconduit au réalisme pur.

Nous pouvons donc résumer de la façon suivante cette comparaison entre Mach et Wittgenstein en ce qui concerne le statut de la subjectivité. Chez ces deux auteurs, on trouve une progression paradoxale depuis une forme d'idéalisme vers une élimination de la subjectivité. Chez Mach, on part de la thèse selon laquelle «le monde n'est constitué que de nos sensations », et c'est cette thèse idéaliste elle-même qui nous conduit paradoxalement à conclure qu'il n'y a rien de tel qu'un sujet pour ces sensations. Chez Wittgenstein, on part de la thèse selon laquelle «je suis mon monde », et c'est cette thèse solipsiste elle-même qui nous conduit paradoxalement à conclure que le Moi n'existe pas, qu'il rétrécit au point de devenir un point sans extension et de ne laisser que le monde en général :

Le monde n'est constitué que de nos sensations

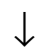

Le Moi peut être élargi au point d'inclure le Monde entier.
Je suis mon monde.

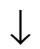

Le Je du solipsisme se réduit à être un point sans étendue.

Dans ces deux cas, donc, on se trouve face à une thèse idéaliste qui nous conduit pourtant à son propre contraire. Demandons-nous à présent comment échapper à une telle difficulté.

\section{Sujet psychologique et sujet métaphysique : « Le Moi se dit en plusieurs sens »}

\section{Lecture mystique ou lecture austère : comment lire le Tractatus ?}

Nous avions vu que, dans le cas de Mach, on hésitait quant à la question de savoir dans quelle mesure sa pensée était une pensée idéaliste, dans quelle mesure au contraire il s'agissait d'une pensée sceptique à l'égard de la notion de sujet. On retrouve donc une même hésitation quand il s'agit d'interpréter la conception de la subjectivité développée à la section 5.6 du Tractatus. Or l'enjeu de cette question est tout à fait majeur. Si, en effet, on lit le Tractatus comme un ouvrage solipsiste, alors on devra adopter une interprétation mystique de la pensée du premier Wittgenstein, lecture selon laquelle la vérité du solipsisme serait une sorte de vérité supérieure dont la caractéristique principale serait de pouvoir être montrée mais non pas dite. Une telle interprétation peut être étayée notamment par certaines propositions des Carnets comme celle qui affirme que « le Je, le Je, voilà le 
profond mystère ! $»^{28} \mathrm{Si}$, au contraire, on choisit de se concentrer sur les propositions qui nous reconduisent au réalisme pur, alors on adoptera une lecture austère du premier Wittgenstein, et on considèrera cette apparente propension au solipsisme comme un simple leurre à visée thérapeutique. L'opposition entre ces deux lectures possibles de la position wittgensteinienne à l'égard du solipsisme n'est donc rien d'autre que l'opposition entre lecture mystique et lecture austère du premier Wittgenstein.

Pourtant, aucune de ces lectures ne semble entièrement satisfaisante : la lecture mystique ne rend pas compte de la façon dont Wittgenstein défend le réalisme pur, tandis que la lecture austère manque d'expliquer l'intérêt incontestable que le premier Wittgenstein porte à la métaphysique solipsiste. C'est pourquoi cette question de savoir si le premier Wittgenstein se faisait l'avocat ou l'ennemi du solipsisme n'a pas de réponse tranchée, comme le fait remarquer notamment David Pears :

On demande si, oui ou non, Wittgenstein était solipsiste quand il a écrit le Tractatus. Si une réponse affirmative implique qu'il acceptait le solipsisme comme une théorie philosophique, alors cette réponse est parfaitement inconciliable avec le texte. D'autre part, si une réponse négative implique qu'il rejette entièrement le solipsisme, c'est là quelque chose qui est exclu par le texte ${ }^{29}$.

Il semble donc que l'on soit, dans cette section du Tractatus, face à une antinomie tout à fait analogue à ces antinomies que Kant présentait dans la Dialectique Transcendantale de sa Critique de la raison pure. Kant y attirait en effet l'attention sur certains problèmes insolubles dans lesquels thèse et antithèse, tout en se contredisant l'une l'autre, étaient individuellement irréfutables. On peut évoquer en particulier la Troisième Antinomie qui posait la question de savoir si le sujet est libre ou s'il est déterminé à l'action par des facteurs causaux ${ }^{30}$ :

\begin{tabular}{|c|c|}
\hline Thèse & Antithèse \\
\hline $\begin{array}{c}\text { «La causalité suivant les lois de } \\
\text { la nature n'est pas la seule d'où } \\
\text { puissent être dérivés les phénomènes } \\
\text { du monde dans leur ensemble. Il est } \\
\text { encore nécessaire d'admettre, pour les } \\
\text { expliquer, une causalité par liberté ». }\end{array}$ & $\begin{array}{c}\text { "Il n'y a pas de liberté mais } \\
\text { des lois de la monde arrive selon }\end{array}$ \\
\hline
\end{tabular}

28. Carnets, 5-8-16.

29. D. Pears: «The Ego and the Eye: Wittgenstein's Use of an Analogy» in Grazer philosophische Studien, 44, 1993, p. 12.

30. Kant, Critique de la raison pure, tr. J.-L. Delamarre et J.-F. Marty, Paris, Gallimard, 1980, p. 408-409. 
De la même manière, la tension inhérente à la question de la subjectivité chez le premier Wittgenstein pourrait prendre la forme de l'antinomie suivante :

\begin{tabular}{|c|c|}
\hline Thèse & Antithèse \\
\hline «Je suis mon monde. (le & $\begin{array}{c}\text { «Il n’y a pas de sujet de la } \\
\text { ensée, de la représentation ». } \\
\text { microcosme) } » .(T L P, 5.631)\end{array}$ \\
\hline
\end{tabular}

Mais on peut alors rappeler la solution qu'apporte Kant à cette antinomie. Celle-ci repose sur une distinction entre deux types de sujet: thèse et antithèse portent en effet respectivement sur un sujet intelligible, inconditionné et libre, et sur un sujet sensible, quant à lui conditionné et déterminé. Et puisque la thèse et l'antithèse ne portent pas sur le même objet, alors elles ne se contredisent pas $^{31}$. Peut-on appliquer une solution analogue à l'antinomie de la section 5.6 ? Peut-on affirmer que, chez Wittgenstein également, thèse solipsiste d'une part et affirmations réalistes d'autre part ne portent pas sur le même aspect de la subjectivité ?

\section{Les deux sujets du Tractatus}

Il semble bien y avoir en effet, dans le Tractatus, deux points de vue possibles sur le sujet. Wittgenstein écrit ainsi à la proposition 5.641 que :

Il y a donc réellement un sens où il peut être question en philosophie d'un je en un sens non psychologique. (...) Le je philosophique n'est ni l'être humain, ni l'âme humaine dont s'occupe la psychologie, mais c'est le sujet métaphysique, qui est frontière - et non partie - du monde ${ }^{32}$.

Ici, Wittgenstein distingue entre, d'une part, un sujet pour le sens commun, un sujet empirique ; et, d'autre part, un sujet dont il peut être question en philosophie, un sujet métaphysique. Par conséquent, quand il défend la primauté du sujet en adoptant une attitude solipsiste, il se réfère au sujet philosophique ; quand, au contraire, il affirme que le sujet disparaît ou rétrécit jusqu'à devenir un point sans extension, il se réfère alors au sujet psychologique ou empirique. Tout comme dans la Troisième Antinomie kantienne, ces deux assertions ne portent donc pas sur le même aspect de la subjectivité et ne se contredisent pas. Examinons alors successivement la nature exacte de ces deux sujets.

31. Kant, op. cit., p. 468.

32. Cette proposition est une synthèse de deux passages des Carnets : à la date du 11-8-16, Wittgenstein envisageait la possibilité de parler du sujet en un sens non psychologique, et à celle du 2-9-16, il soulignait que le je philosophique n'était ni l'homme ni son corps ni son âme mais le sujet métaphysique qui est frontière et non partie du monde. 
On peut commencer par le sujet psychologique. Celui-ci correspond au sujet empirique, au sujet de la représentation et même à l'âme en général. Le premier Wittgenstein, à l'exemple de Mach, entreprend sans équivoque d'éliminer ce sujet psychologique. Pourtant, une telle élimination n'a pas pour fonction de montrer que ce sujet n'a pas de réalité $d u$ tout : elle vise plutôt à montrer que ce sujet n'existe pas comme substance simple. Selon Wittgenstein, le sujet de la représentation est un simple fait doué de la même complexité que la réalité qu'il se représente ${ }^{33}$. Affirmer comme il le fait qu'il n'y a «pas de sujet de la représentation », c'est donc affirmer que la seule chose qui existe, c'est un complexe composé d'idées et de pensées mais dépourvu d'unité propre. En revanche, l'âme simple et indivisible telle que se la représentait la métaphysique traditionnelle ne peut être considérée que comme une «pseudo-chose », une monstruosité (ein Unding) ${ }^{34}$. En d'autres termes, Wittgenstein réfute ici la métaphysique classique en montrant que l'âme n'existe pas, et il réfute la psychologie empirique en montrant que le prétendu sujet de la pensée n'est pas véritablement un sujet mais au mieux un complexe de représentations.

Pourtant, ce sujet psychologique n'est pas, selon Wittgenstein, le seul sujet. Bien au contraire, comme le souligne Sebastian Lalla, «l'effondrement du sujet empirique fait immédiatement signe vers le sujet métaphysique ${ }^{35}$. Il convient par conséquent de se demander ce qui est exactement désigné par cette expression de «sujet métaphysique». Assurément, quand Wittgenstein emploie ici le qualificatif de "métaphysique", il ne se réfère nullement au sujet de la métaphysique classique : bien au contraire, ce terme renvoie ici au fait que le sujet en question est «limite, et non partie, du monde ${ }^{36}$. On comprend alors pourquoi Wittgenstein affirmait qu' «en un sens important il n'y a pas de sujet » : ce sujet n'existe nulle part au sein de la réalité $e^{37}$, parce que la limite n'est pas incluse dans ce qu'elle limite. Mais affirmer qu'en un sens important il n'y a pas de sujet, ce n'est pas dire qu'il n'y a pas de sujet du tout: car dans un autre sens, non moins important, le sujet métaphysique s'avère également être une limite de ce monde duquel il est absent.

33. Cf. TLP, 5.542 : «Il est cependant clair que "A croit que p", “A pense que p", "A dit p" sont de la forme ""p" dit p", et il ne s'agit pas ici de la coordination d'un fait et d'un objet, mais de la coordination de faits par la coordination de leurs objets ».

34. Cf. TLP, 5.5421 : «Ceci montre encore que l'âme - le sujet, etc. - telle qu'elle est conçue dans la psychologie superficielle d'aujourd'hui, est une pseudo-chose. Car une âme composée ne serait plus une âme ».

35. S. Lalla: Solipsismus bei Ludwig Wittgenstein, eine Studie zur Früh- und Spätwerk, Wittgenstein-Studien, 4, 2002, p. 35.

36. TLP, 5.641 .

37. Ibid., 5.633 : « Où, dans le monde, un sujet métaphysique peut-il être discerné ?» 


\section{Le sujet métaphysique : sujet transcendantal ou porteur de l'éthique ?}

Dans ces conditions, il serait tentant d'identifier ce sujet métaphysique wittgensteinien au sujet transcendantal kantien. Chez Kant, le sujet transcendantal est une condition de possibilité et du monde et de l'unité des représentations. En va-t-il de même avec le sujet métaphysique de Wittgenstein? Une telle lecture est défendue par un courant classique d'interprétation du premier Wittgenstein ${ }^{38}$, et elle pourrait en effet être appuyée par certains passages des Carnets 1914-1916 où l'on peut lire, par exemple, que «le sujet n'est pas une partie du monde mais une présupposition de son existence ${ }^{39}$. Pourtant, cette interprétation ne saurait être acceptée sans réserve. Si, en effet, Wittgenstein qualifie bien sa position de $«$ transcendantale ${ }^{40}$, cela n'implique pas qu'il emploie ce terme au sens où l'emploie Kant. Bien au contraire, il y a une différence considérable entre transcendantalisme kantien et transcendantalisme wittgensteinien. Cette différence est en particulier soulignée par Jacques Bouveresse dans Le mythe de l'intériorité, où il montre que tandis que le sujet transcendantal kantien était le sujet d'un «Je pense » qui «doit pouvoir accompagner toutes mes représentations », le sujet métaphysique de Wittgenstein est quant à lui si évanescent, si dépourvu d'extension qu'il ne saurait pas même se saisir dans un « Je pense » :

Le Moi philosophique wittgensteinien est un «Je», mais il n'est pas véritablement un «Je pense ». (...) Il est bien, si l'on veut, une condition de possibilité de l'expérience en général, mais en un sens encore beaucoup plus formel (...) que chez Kant ${ }^{41}$.

On peut donc reposer le problème de la nature du sujet métaphysique chez Wittgenstein de la façon suivante : $1^{\circ}$ malgré son élimination du sujet empirique, Wittgenstein maintient la réalité d'un sujet philosophique qui, quant à lui, ne saurait être éliminé $; 2^{\circ}$ ce sujet est un sujet métaphysique ou transcendantal. Cependant, $3^{\circ}$ il n'est pas transcendantal en un sens kantien. En quel sens, alors, ce sujet est-il transcendantal ? Nous allons à présent conclure cet article en soutenant que ce sujet est transcendantal en un sens éthique.

Nous avons jusqu'à présent examiné le sujet empirique et le sujet métaphysique. Mais il est un autre aspect de la subjectivité que nous ne saurions laisser de côté : parler du sujet, c'est également parler du sujet de la volonté. Que nous dit Wittgenstein à propos de ce sujet qu'il présente

38. Voir en particulier l'interprétation défendue par Erik Stenius dans son ouvrage intitulé Wittgentein's Tractatus, a Critical Exposition of its Main lines of Thought, Oxford, Blackwell, 1960.

39. Carnets, 2-8-16.

40. TLP, 6.13 : «La logique est transcendantale».

41. J. Bouveresse : Le Mythe de l'intériorité, Expérience, sensation et langage privé chez Wittgenstein, Paris, Minuit, 1987, p. 167. 
comme le «porteur de l'éthique ${ }^{42}$ ? Tout comme il distinguait entre subjectivité empirique et philosophique en général, Wittgenstein établit une distinction entre volonté empirique et volonté métaphysique. Il se réfère en effet, dans les Carnets, à une volonté psychologique consistant dans l'ensemble des impulsions et motifs susceptibles de déterminer l'action et de «mouvoir le corps humain ${ }^{43}$. Cette volonté empirique est donc l'équivalent, dans le domaine de la volonté, du sujet psychologique dont nous avons parlé plus haut. Pourtant, cette volonté ne constitue pas l'intégralité de la volonté : elle s'oppose au contraire à une volonté métaphysique qui sera à son tour l'expression de la subjectivité métaphysique sur le plan de la volonté.

Or cette volonté métaphysique peut être quant à elle être à juste titre qualifiée de volonté transcendantale. En effet, elle est bien une condition de possibilité : non plus du monde mais de ses valeurs. Ainsi, le monde est, en lui-même, éthiquement indifférent. C'est pourquoi les valeurs éthiques ne sauraient être fixées que par un sujet qui le considère depuis un point de vue supérieur, depuis ce point de vue transcendantal qui est celui de la volonté métaphysique. Selon Wittgenstein, «le bien et le mal n'apparaissent que par le sujet ${ }^{44}$ : or cette autorité éthique n'est autre que le sujet transcendantal de la volonté. On comprend alors en quel sens le sujet wittgensteinien est transcendantal : il l'est en tant que sujet d'une volonté éthique.

Dans ces conditions, en quoi consiste le devoir proprement éthique qui est imposé aux sujets ? Ce devoir est celui d'opérer une conversion de la volonté depuis son état empirique vers son état philosophique ou transcendantal. C'est à une telle conversion que Wittgenstein fait allusion à la proposition $6.43 \mathrm{du} T L P$ :

Si le bon ou le mauvais vouloir changent le monde, ils ne peuvent changer que les frontières du monde, non les faits (...). En bref, le monde doit alors devenir par-là totalement autre. Il doit pouvoir, pour ainsi dire, diminuer ou croître dans son ensemble.

Le monde de l'homme heureux est un autre monde que celui de l'homme malheureux.

Ce changement de perspective qui transforme le monde de l'homme malheureux en monde de l'homme heureux est le pur et simple effet de la volonté philosophique/métaphysique. La différence entre ces deux mondes n'est en effet nullement une différence factuelle. Elle ne dépend que de la façon dont le sujet s'accorde ou non avec son monde: et un tel consentement est l'effet de la volonté transcendantale. C'est pourquoi le seul impératif catégorique de la philosophie de Wittgenstein est contenu dans la

42. $T L P, 6.423$.

43. Carnets, 14-7-16.

44. Ibid., 2-8-16. 
formule suivante : «Sois heureux ! ${ }^{45}$. Ce bonheur est réalisé par la volonté métaphysique : il témoigne de ce que le sujet empirique ordinaire, par cette conversion de sa volonté, est devenu un «homme digne ».

Récapitulons à présent les conclusions que nous pouvons tirer de cette étude. Chez Mach, nous sommes confrontés au paradoxe d'une philosophie apparemment idéaliste qui est pourtant indissociable d'une élimination de ce Moi qu'elle aurait pourtant dû promouvoir. Ce paradoxe trouve un écho dans la première philosophie de Wittgenstein : ici aussi, une attitude solipsiste se voit associée à une critique sceptique de la notion de sujet. Pourtant, bien que Wittgenstein se fasse le continuateur de la critique machienne du sujet empirique, il n'en va pas de même avec le sujet métaphysique ou philosophique. Ce sujet en un sens non psychologique est assurément absent de l'expérience, mais pour des raisons bien spécifiques : parce qu'il est une limite de l'expérience qui en constitue la condition de possibilité. Il semble donc qu'à la différence de Mach qui proposait une élimination unilatérale du sujet, le premier Wittgenstein propose quant à lui une requalification de la subjectivité. En cela, il renvoie dos-à-dos le scepticisme qui refuse d'attribuer au sujet une quelconque réalité, et le dogmatisme qui lui confère une réalité absolue. Le sujet philosophique est chez lui l'expression d'un tel compromis : un sujet qui n'appartient pas au monde et qui cependant, en le limitant, en est une condition de possibilité ; un sujet qui n'est pas un objet pour la psychologie et qui, cependant, en est un pour la philosophie. Par conséquent, il semble qu'en réintroduisant ce sujet philosophique, le premier Wittgenstein soit parvenu à trouver une troisième voie entre la métaphysique cartésienne d'une part, les thèses éliminativistes de Mach de l'autre. C'est cette position de subtil compromis entre dogmatisme et scepticisme qui peut donner l'impression d'une oscillation permanente du Tractatus : elle n'en est pas moins porteuse d'une profonde cohérence de la philosophie du premier Wittgenstein. 


\section{Bibliographie sélective}

Anscombe G.E.M., An Introduction to Wittgenstein's Tractatus, Themes in the Philosophy of Wittgenstein, London, Hutchinson, 1971.

Bell D., «Solipsism and Subjectivity», The European Journal of Philosophy, 4 (2), p. 155-174.

Blackmore J., Ernst Mach, his Life and Influence, Berkeley, University of California Press, 1972.

Bouveresse J., Le Mythe de l'intériorité, Expérience, sensation et langage privé chez Wittgenstein, Paris, Minuit, 1987.

Coyne M. U., «Eye, I and Mine : the Self of Wittgenstein's Tractatus », The Southern Journal of Philosophy, 20,1982, p. 313-323.

Floyd J., «The Uncaptive Eye : Solipsism in Wittgenstein's Tractatus », in L.S. Rouner (dir.), Loneliness, Notre-Dame, Notre-D. UP, 1998.

Gabriel G., «Solipsismus: Wittgenstein, Weininger und die Wiener Moderne », in H. Bachmaier (dir.), Paradigmen der Moderne, Amsterdam, J. Benjamin, 1990.

Haag J., « Der Wille als Träger der Ethik », Acta Analytica, 13, 1995, p. 131-152.

Haller R., «Ernst Mach : das unrettbare Ich», in J. Speck (dir.), Grundprobleme der großen Philosophen, Göttingen, Vandenhoeck, 1991.

Haller R., "Bemerkungen zur Egologie Wittgensteins », in Wittgenstein in Focus, im Brennpunkt Wittgenstein, dir. B. McGuinness et R. Haller, Amsterdam, Rodopi, 1989.

Haller R., «Unklarheiten über das ich oder,Ich', Ludwig Wittgenstein », Revue internationale de philosophie, 169, 69, p. 249-263.

Haller R. et Stadler F., Ernst Mach, Werk und Wirkung, Vienne, HölderPichler-Tempsky, 1988.

Hamilton A., «Ernst Mach and the Elimination of Subjectivity », Ratio, 1990, p. 117-135.

Hintikka J., « On Wittgenstein's Solipsism », Mind, 67, 1958, p. 88-91.

Kant, Critique de la Raison pure, tr. J.-L. Delamarre et J.-F. Marty, Paris, Gallimard, 1980.

Laugier S., «La question d'une subjectivité sans psychologie », Europe, 906, 2004, p. 106-120.

Lalla S, «Solipsismus bei Ludwig Wittgenstein, eine Studie zur Früh- und Spätwerk », Wittgenstein-Studien, 4, 2002.

Le Rider J., Modernité viennoise et crises de l'identité, Paris, PUF, 1990. 
Mach, Erkenntnis und Irrtum, Skizzen zu eine Psychologie der Forschung, Leipzig, 1905. - Tr. fr., M. Dufour, La Connaissance et l'erreur, Paris, Flammarion, 1919.

Mach, Die Analyse der Empfindungen, Iena, G. Fischer, 1886, tr. F. Eggens et J.-M. Monnoyer : L'Analyse des sensations, Nîmes, J. Chambon, 1996.

Pears D., "The Ego and the Eye: Wittgenstein's Use of an Analogy», Grazer philosophische Studien, 44, 1993, p. 59-68.

Pears D., «Was the Early Wittgenstein a Solipsist? », Wittgenstein-Studien, 2, 2001, p. 13-22.

Pears D., The False Prison, a Study of the Development of Wittgenstein's Philosophy, Oxford, Oxford UP, 1987 - Tr. Ch. Chauviré, La penséeWittgenstein, Paris, Aubier, 1993.

Sackur J., "Théorie de la connaissance et solipsisme des Remarques philosophiques au Cahier Bleu», in Wittgenstein : les mots de l'esprit, dir. Ch. Chauviré, S. Laugier et J.-J. Rosat, Paris, Vrin, 2002.

Schulte J., "The Key to Solipsism », in J. Hintikka et K. Puhl (dir.), The British Tradition in the twentieth Century, Amsterdam, Rodopi, 1993.

Sluga H., «Subjectivity in the Tractatus », Synthese, 56, 1983, p. 123-129.

Soulez A., «Du "moi dissous" à la méthode scientifique. De la reconstruction de l'égologie », Philosophia Scientiae, 3 (2), 1998, p. $142-154$.

Stenius E., Wittgentein's Tractatus, a Critical Exposition of its Main lines of Thought, Oxford, Blackwell, 1960.

Wittgenstein, Carnets 1914-1916, tr. G.-G. Granger, Paris, Gallimard, 1971.

Wittgenstein, Tractatus logico-philosophicus, Frankfurt, Suhrkamp, 1999 ; tr. fr. G.-G. Granger, Paris, Gallimard, 1993.

Wittgenstein, Remarques philosophiques, tr. J. Fauve, Paris, Gallimard, 1975. 